\title{
Evaluating the Burden of Caregivers in Children with Developmental Coordination Disorder in Indian Context
}

\author{
Ganapathy Sankar $\mathbf{U}^{1}$ and Monisha $\mathbf{R}^{2 *}$ \\ ${ }^{1}$ Professor, SRM College of Occupational Therapy, India \\ ${ }^{2}$ Assistant Professor, SRM Institute of Science and Technology, India \\ *Corresponding author: Monisha R, Assistant Professor, SRM College of Physiotherapy, SRM Institute of Science and Technology, Chennai, India
}

Submission: 眥 August 25, 2018; Published: 阱 October 15, 2018

\begin{abstract}
Introduction: Children with DCD faces complex hindrance in self-care functions and in all the activities of daily living. Caregiver is parent or the sole guardian, who took part in the growth and wellness of children with developmental coordination disorder.
\end{abstract}

Objective: Objective of the study is to assess the caregiver's burden in handling children with developmental coordination disorder (DCD).

Methodology: A convenience sample of 10 children with DCD (6 girls and 4 boys) was enrolled. Previous researchers have documented that caregivers of children with developmental disability ends with depression. Beck Depression Inventory (BDI) was used to identify the psychological impact of caregivers of these children with DCD

Discussion: For the development and care of child with DCD, there is a need for maximum care and attention when compared to the care provided for the children of normally developing age. However, their exist a hypothesis that most parents adapt to the caring process of the children with coordination disorder. Care givers well-being and general health were not given importance and in daily routine they experience fatigue and boredom

Conclusion: Caregivers of children with DCD experience a burden and there is a need for psychological counselling and relaxation training and further studies are planned to follow up these parents of children with DCD to devise appropriate treatment protocol and therapeutic intervention

Keywords: Caregivers; Burden; DCD; CHQ-50; BDI

\section{Introduction}

Care for children with developmental coordination disorder is the most important task that needs to be provided by the family and the caregivers of the children to establish the successful treatment strategies. Care and providing the appropriate timely treatment facilitate these children towards progress. Patterns of diseases that affects children various in dimensional variety and in 2018, the most predominant disorder that confronts children in developing as well as developed countries is DCD [1]. Developmental coordination disorder is a chronic and usually a permanent condition that affects the motor coordination of children. These children suffer because of the motor coordination difficulties and have poor academic performance, that affects their education and continuing education to higher secondary level is almost an impossible task for these children [2].

Normally developing children enjoys a healthy childhood day with little need for health care services. But children with DCD experience difficulties in childhood and have frequent visit to health care center and they have increased demand in utilization of health care services, over time these children will become dependent on variable treatment strategies [3]. Motor impairment is the hallmark feature of DCD. These children have predominant symptoms like deficit in sensory integration, communication as because of poor handwriting and language skills. These children instead of having higher IQ of greater than $70 \%$ faces difficulty in planning and sequencing the activity of daily living [4]. Children with DCD faces complex hindrance in self-care functions and in all the activities of daily living. Caregiver is parent or the sole guardian, who took part in the growth and wellness of children with developmental coordination disorder. When these children experience functional limitation and faces difficulty in performing motor tasks, it takes the role of their caregivers in assisting these children and motivating them to perform and acquire the motor skills which are needed to accomplish the task [5]. 
Children with DCD will have trouble in ADL and extensive caregiving is the utmost need for these children. Each children's caregiver will have a varying psycho social illness that hinders the growth and well-being of the children diagnosed as DCD. Developing timely management and diagnosis of DCD will maximizes the prognosis of children with motor coordination difficulties [6]. Caregivers spends their time and money on the diagnosis of these childhood motor deficit and in deciding the appropriate treatment protocol. When the children experience functional limitations and long-term dependence, it takes the responsibility of caregivers to maximize their care towards the children with DCD. Caregivers will have psycho-social impact when they assist children with DCD at their problems in movement inhibition and sequencing of motor task [7].

\section{Methodology}

After getting informed consent from the parents regarding their willingness to participate in this study and the ethical clearance has been obtained by the institutional ethical committee. Baseline evaluation of children with DCD has been assessed using Bruininks-Oseretsky Test of Motor Proficiency-Second Edition, after baseline evaluation, caregivers of those children with DCD has been evaluated with Child Health Questionnaire-Parent Form 50. Objective of the study is to assess the caregiver's burden in handling children with developmental coordination disorder (DCD). A convenience sample of 10 children with DCD 6 girls and 4 boys) was enrolled. Previous researchers have documented that caregivers of children with developmental disability ends with depression. Beck Depression Inventory (BDI) was used to identify the psychological impact of caregivers of these children with DCD. Children with DCD had significantly poorer performance in all the activity of daily living. Lower HRQOL is documented in all domains of physical activity in children with DCD, hence they rely on their parents for all their basic need [8].

\section{Data Collection}

Table 1-4 and Figure 1,2

Table 1: Frequency table for child health questionnaire-parent form 50 global health.

\begin{tabular}{|c|c|c|c|c|c|}
\hline \multicolumn{6}{|c|}{ Global Health } \\
\hline & & Frequency & Percent & Valid Percent & Cumulative Percent \\
\hline \multirow[t]{3}{*}{ Valid } & 4 & 1 & 10 & 10 & 10 \\
\hline & 5 & 9 & 90 & 90 & 100 \\
\hline & Total & 10 & 100 & 100 & \\
\hline \multicolumn{6}{|c|}{ Physical Activity } \\
\hline \multirow[t]{3}{*}{ Valid } & 1 & 6 & 60 & 60 & 60 \\
\hline & 2 & 4 & 40 & 40 & 100 \\
\hline & Total & 10 & 100 & 100 & \\
\hline \multicolumn{6}{|c|}{ Everyday Activity } \\
\hline \multirow[t]{3}{*}{ Valid } & 1 & 7 & 70 & 70 & 70 \\
\hline & 2 & 3 & 30 & 30 & 100 \\
\hline & Total & 10 & 100 & 100 & \\
\hline \multicolumn{6}{|c|}{ Pain } \\
\hline \multirow[t]{4}{*}{ Valid } & 4 & 2 & 20 & 20 & 20 \\
\hline & 5 & 7 & 70 & 70 & 90 \\
\hline & 6 & 1 & 10 & 10 & 100 \\
\hline & Total & 10 & 100 & 100 & \\
\hline \multicolumn{6}{|c|}{ Behaviour } \\
\hline \multirow[t]{3}{*}{ Valid } & 2 & 3 & 30 & 30 & 30 \\
\hline & 3 & 7 & 70 & 70 & 100 \\
\hline & Total & 10 & 100 & 100 & \\
\hline \multicolumn{6}{|c|}{ Well Being } \\
\hline \multirow[t]{3}{*}{ Valid } & 1 & 4 & 40 & 40 & 40 \\
\hline & 2 & 6 & 60 & 60 & 100 \\
\hline & Total & 10 & 100 & 100 & \\
\hline \multicolumn{6}{|c|}{ Self Esteem } \\
\hline
\end{tabular}




\begin{tabular}{|c|c|c|c|c|c|}
\hline Valid & 4 & 3 & 30 & 30 & 30 \\
\hline & 5 & 7 & 70 & 70 & 100 \\
\hline & Total & 10 & 100 & 100 & \\
\hline \multicolumn{6}{|c|}{ Your Child Health } \\
\hline \multirow[t]{3}{*}{ Valid } & 1 & 7 & 70 & 70 & 70 \\
\hline & 2 & 3 & 30 & 30 & 100 \\
\hline & Total & 10 & 100 & 100 & \\
\hline \multicolumn{6}{|c|}{ You and your Child } \\
\hline \multirow[t]{3}{*}{ Valid } & 4 & 4 & 40 & 40 & 40 \\
\hline & 5 & 6 & 60 & 60 & 100 \\
\hline & Total & 10 & 100 & 100 & \\
\hline \multicolumn{6}{|c|}{ Facts about your Child } \\
\hline \multirow[t]{3}{*}{ Valid } & 1 & 4 & 40 & 40 & 40 \\
\hline & 2 & 6 & 60 & 60 & 100 \\
\hline & Total & 10 & 100 & 100 & \\
\hline \multicolumn{6}{|c|}{ Facts About You } \\
\hline \multirow[t]{3}{*}{ Valid } & 1 & 4 & 40 & 40 & 40 \\
\hline & 2 & 6 & 60 & 60 & 100 \\
\hline & Total & 10 & 100 & 100 & \\
\hline
\end{tabular}

Table 2: Beck depression inventory (BDI).

\begin{tabular}{|c|c|c|c|}
\hline N-total Number of Children with DCD & Caregivers Gender & Gender of the Child with DCD & Beck Depression Inventory (BDI) \\
\hline 1 & M & B & 30 \\
\hline 2 & F & B & 39 \\
\hline 3 & M & B & 35 \\
\hline 4 & F & B & 27 \\
\hline 5 & M & G & 40 \\
\hline 6 & F & G & 45 \\
\hline 7 & F & G & 40 \\
\hline 8 & F & G & 40 \\
\hline 9 & F & G & 42 \\
\hline 10 & M & G & 4 \\
\hline
\end{tabular}

Table 3: Back depression inventory BDI.

\begin{tabular}{|l|c|c|c|c|c|}
\hline & & Frequency & Percent & Valid Percent & Cumulative Percent \\
\hline Valid & Moderate depression & 4 & 40 & 40 & 40 \\
\hline & Severe depression & 6 & 60 & 60 & 100 \\
\hline & Total & 10 & 100 & 100 & \\
\hline
\end{tabular}

Table 4: Back depression inventory BDI and gender difference of care giver.

\begin{tabular}{|l|c|c|c|}
\hline & & \multicolumn{2}{|c|}{ Gender of Care Giver } \\
\hline \\
Back Depression Inventory BDI & Moderate Depression & Male & Female \\
\hline
\end{tabular}




\begin{tabular}{|c|c|c|c|c|}
\hline & Severe Depression & 0 & 6 & 6 \\
\hline Total & & 4 & 6 & 10 \\
\hline
\end{tabular}

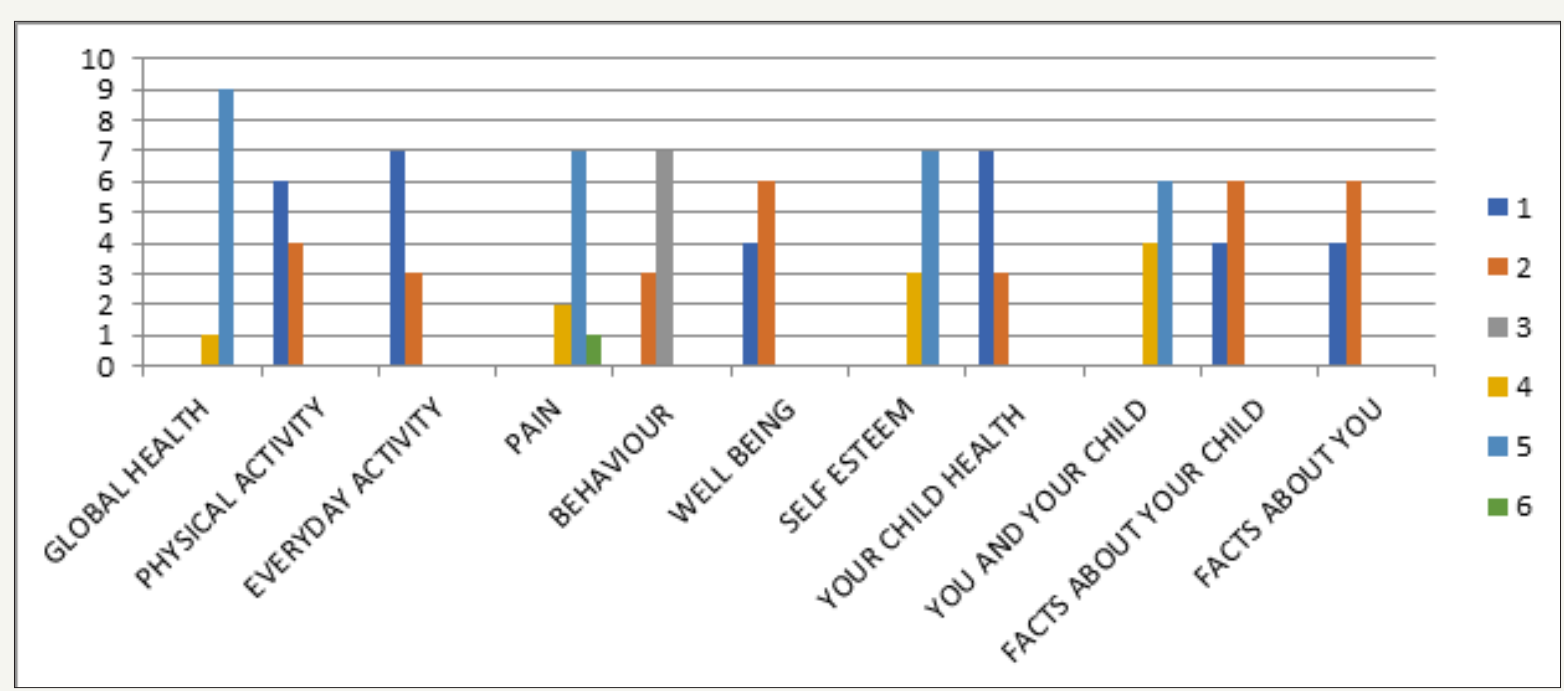

Figure 1

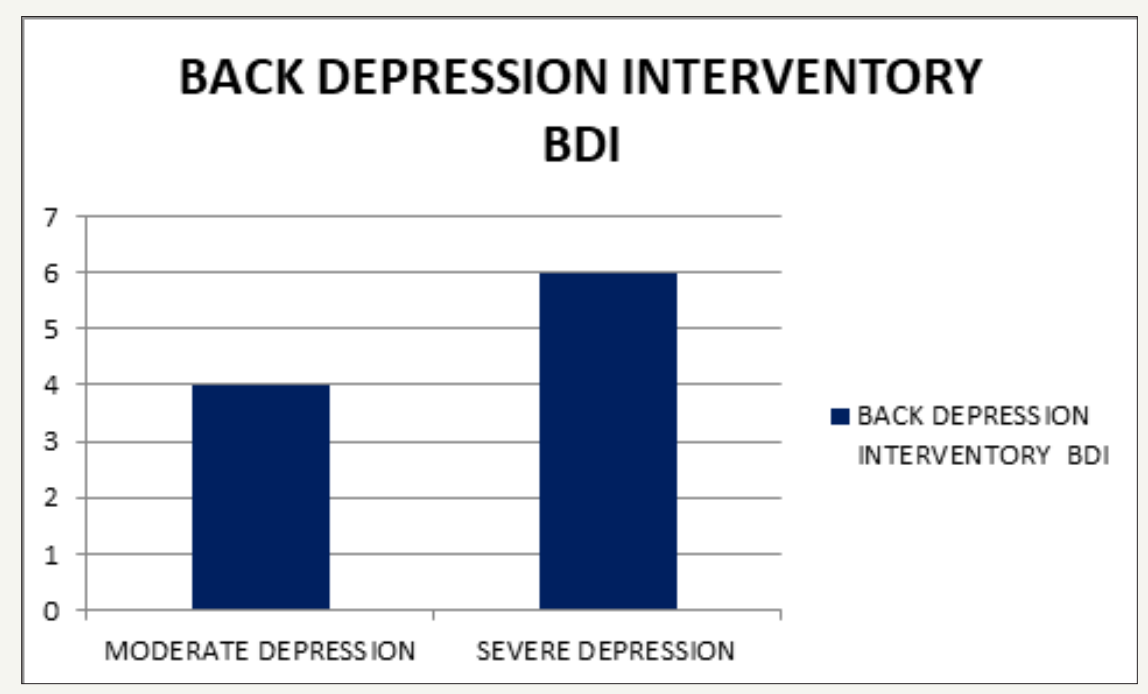

Figure 2

\section{Discussion}

Care for children with DCD is the utmost responsibility of the parents. For the development and care of child with DCD, there is a need for maximum care and attention when compared to the care provided for the children of normally developing age. However, them exist a hypothesis that most parents adapt to the caring process of the children with coordination disorder. Care givers well-being and general health were not given importance and in daily routine they experience fatigue and boredom, and this affects their general health and well-being. Psycho-somatic disorders will accompany with caregivers of the children with developmental coordination disorder. Evidence has if caregivers of these children experiences considerable variations in the attitude of care provided. There exist wide-variation in the care, because of the gender and cultural difference. Hence the current study was done to highlight the burden of caregivers of children with DCD. A detailed examination of global health, physical activity, everyday activity, behavior, well-being, self-esteem, child's health as assumed by the parent, child and the parent relation, facts about the child and facts about the parent were examined in detail and the gender and cultural difference has been given a special attention [9]. The most important assessor of caregiver's burden is child behavior and physical activity of the child. Highest level of depression has been reported in the parents of children with DCD. When the gender difference has been considered, it has been proved that parents of the child, especially mothers were affected with severe depression as compared to the fathers. 


\section{Conclusion}

The psychological and physical health of caregivers is evaluated in detail using CHQ-50 and it has been reported that caregivers were facing extreme depression by taking of the children with DCD, in this study it has been proved that primarily mothers were affected with depression, was strongly influenced by child behavior and caregiving demands [10]. Child behavior problems were an important predictor of caregiver well-being. The day-to-day activity of daily living of the children with DCD created challenges for parents for providing their utmost care. Further follow up of these caregivers were planned to deliver therapeutic intervention and psychological counselling. .

\section{References}

1. Ganapathy U, Saritha S (2011) A study of prevalence of developmental coordination disorder (DCD) at Kattankulathur, Chennai, Indian Journal of Physiotherapy and occupational therapy 5(1): 63-65.

2. Ganapathy U (2018) The prevalence of developmental coordination disorder at Kattupakkam, Tamilnadu. IOSR Journal of Pharmacy 8(2): 49-52.

3. Zattara M, Bouisset S (1988) Posturo-kinetic organisation during the early phase of upper limb movement. J Neurol Neurosurg Psychiatry 51(7): 956-965.
4. Williams H, Fisher J, Tritschler K (1983) Descriptive analysis of static postural control in 4, 6 and 8 year old normal and motorically awkward children. Am J Phys Med 62(1): 12-26.

5. Van Dellen T, Geuze R (1988) Motor response processing in clumsy children. J Child Psychol Psychiatry 29(4): 489-500.

6. Shumway-Cook A, Woollacott MH (1985) The growth of stability: postural control from a developmental perspective. J Mot Behav 17(2): 131147.

7. Pal_tsev Y, El_ner A (1967) Preparatory and compensatory period during voluntary movement in patients with involvement of the brain at different locations. Pflugers Archives 12: 161-168.

8. Cairney J, Hay JA, Faught BE, Wade TJ, Corna L, et al. (2005) Developmental coordination disorder, generalized self-efficacy toward physical activity, and participation in organized and free play activities. J Pediatr 147(4): 515-520.

9. Mcllroy WE, Maki BE (1997) Preferred placement of the feet during quiet stance: development of a standardized foot placement for balance testing. Clin Biomech 12(1): 66-70.

10. Sibella F, Galli M, Romei M, Montesano A, Crivellini M (2003) Biomechanical analysis of sit-to-stand movement in normal and obese subjects. Clin Biomech 18(8): 745-750.
Creative Commons Attribution 4.0 International License

For possible submissions Click Here

\section{Submit Article}

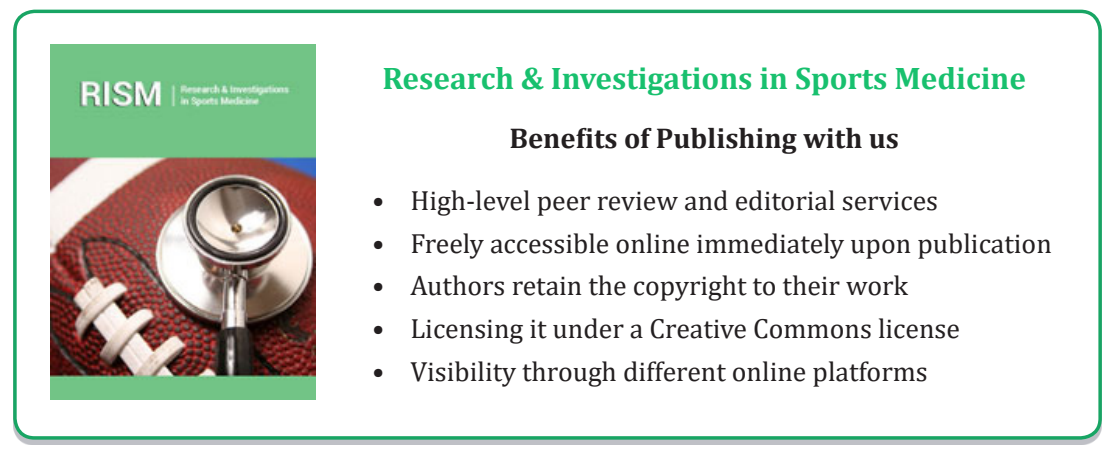

